\title{
Minimal impact of selective susceptibility reporting on the use of piperacillin-tazobactam for Escherichia coli and Klebsiella bacteremia
}

\author{
Yorgo Zahlanie $\mathrm{MD}^{1}$, Brenton C. Hall PharmD², Wenjing Wei PharmD ${ }^{1,2}$, Norman S. Mang PharmD ${ }^{1,2}$, \\ Jessica K. Ortwine PharmD ${ }^{1,2}$, Shelby Anderson PharmD², Kristi Morgan MT (ASCP)², Tamara P. Guzman BS MT III (ASCP)2, \\ Linda S. Hynan $\mathrm{PhD}^{1}$ and Bonnie C. Prokesch MD ${ }^{1,2}$ (1) \\ ${ }^{1}$ University of Texas Southwestern Medical Center, Dallas, Texas and ${ }^{2}$ Parkland Health and Hospital System, Dallas, Texas
}

\begin{abstract} blood cultures with either Escherichia coli or Klebsiella spp.

Antimicrobial resistance has resulted in $>2.8$ million infections and 35,000 deaths annually in the United States as well as increased hospital length of stay (LOS) and high costs. ${ }^{1,2}$ Multiple antimicrobial stewardship interventions have been suggested to improve antibiotic use, slow resistance development resistance, and minimize adverse events. Selective reporting, as a stewardship strategy, involves not reporting antibiotic susceptibilities to providers when treatment is not indicated, preferentially reporting narrow-spectrum agents, or excluding specific antibiotics from the report to discourage use. ${ }^{3}$ Although the Infectious Diseases Society of America recommends selective reporting as one possible stewardship intervention, the weak recommendation is based on low-quality evidence. ${ }^{4}$ Considering the potential for selective reporting to improve antibiotic utilization, patient outcomes, and susceptibility rates as well as the paucity of data in this area, we studied the effect of selective reporting on antibiotic de-escalation.
\end{abstract}

Selective cascade reporting of antibiotic susceptibilities did not have a significant impact on de-escalation from piperacillin-tazobactam (PT), duration of PT use, length of stay, or rates of acute kidney injury and Clostridioides difficile infection in patients with positive monomicrobial

(Received 8 August 2020; accepted 10 December 2020; electronically published 11 January 2021) Secondary objectives included impact on the duration of PT use,

\section{Methods}

Antimicrobial utilization data from our institution in 2016 revealed that piperacillin-tazobactam (PT) was the most commonly prescribed broad-spectrum gram-negative agent. In addition, review of microbiology data in 2016 identified $>13,000$ gram-negative bacterial isolates, of which $>85 \%$ were Escherichia coli and Klebsiella spp. Because of the overwhelming number of infections with E. coli and Klebsiella spp and the fact that treatment does not generally require $\mathrm{PT}$ at our institution where $>80 \%$ of isolates are susceptible to ceftriaxone, we

Author for correspondence: Bonnie C. Prokesch, E-mail: Bonnie.Prokesch@ UTSouthwestern.edu

Cite this article: Zahlanie Y, et al. (2021). Minimal impact of selective susceptibility reporting on the use of piperacillin-tazobactam for Escherichia coli and Klebsiella bacteremia. Infection Control \& Hospital Epidemiology, 42: 1272-1274, https://doi.org/ 10.1017/ice.2020.1385 focused on the impact of selective reporting for E. coli and Klebsiella in blood cultures on the use of PT. The primary objective of this study was to determine the impact of selective reporting on the de-escalation rate from PT at any time during the hospital stay in patients with E. coli and Klebsiella bacteremia. LOS, and rates of acute kidney injury (AKI) and Clostridioides difficile infection (CDI).

We performed a retrospective chart review of hospitalized patients aged $\geq 18$ years with monomicrobial bacteremia secondary to non-extended-spectrum $\beta$-lactamase (ESBL)-producing E. coli or Klebsiella isolates between March 7, 2016, and March 7, 2018. Exclusion criteria were polymicrobial blood cultures with either E. coli and/or Klebsiella in addition to another organism not deemed to be a contaminant and discontinuation of PT before release of antibiotic susceptibilities. The data are outlined in Table 1. Antibiotics considered to be a de-escalation from PT were aminopenicillins, first-, second- or third-generation cephalosporins, oral fluoroquinolones, oral trimethoprim-sulfamethoxazole, and nitrofurantoin. AKI was defined as an increase in serum creatinine by $\geq 50 \%$ from the lowest serum creatinine while on PT or as an absolute increase of $\geq 0.3 \mathrm{mg} / \mathrm{dL}$ between 2 consecutive values while on PT according to the Acute Kidney Injury Network classification system. ${ }^{5}$

A new cascaded susceptibility reporting algorithm applicable to non-ESBL E. coli and Klebsiella isolates was implemented on September 7, 2017 (Fig. 1). Notably, clinicians were able to request the release of any suppressed antibiotic susceptibility at any time. Moreover, PT is not a restricted antibiotic that is regularly audited by the stewardship team at our institution; thus, this intervention was not accompanied by generalized audit and feedback.

The Mann-Whitney U test was used for continuous variables, while the $\chi^{2}$ test or Fisher exact test was used for categorical variables. The significance level was set at $P<.05$. The statistical

(C) The Author(s), 2021. Published by Cambridge University Press on behalf of The Society for Healthcare Epidemiology of America. This is an Open Access article, distributed under the terms of the Creative Commons Attribution-NonCommercial-NoDerivatives licence (http://creativecommons.org/licenses/by-nc-nd/4.0/), which permits non-commercial re-use, distribution, and reproduction in any medium, provided the original work is unaltered and is properly cited. The written permission of Cambridge University Press must be obtained for commercial re-use or in order to create a derivative work. 
Table 1. Patient Characteristics and Outcomes

\begin{tabular}{|c|c|c|c|}
\hline Characteristic & $\begin{array}{l}\text { Preintervention } \\
\text { Period }\end{array}$ & $\begin{array}{l}\text { Postintervention } \\
\text { Period }\end{array}$ & $P$ Value \\
\hline Sample size, no. & 201 & 174 & \\
\hline Age, median y & 52.7 & 54.7 & .322 \\
\hline Sex, female, no. (\%) & $112(55.7)$ & 99 (56.9) & .819 \\
\hline \multicolumn{4}{|l|}{ Race, no. (\%) } \\
\hline Hispanic & $133(66.2)$ & $110(63.2)$ & \multirow[t]{4}{*}{.022} \\
\hline Black & $44(21.9)$ & $31(17.8)$ & \\
\hline White & $15(7.5)$ & $30(17.2)$ & \\
\hline Other & $9(4.5)$ & $3(1.7)$ & \\
\hline \multicolumn{4}{|l|}{$\begin{array}{l}\text { Organism speciated, } \\
\text { no. }(\%)\end{array}$} \\
\hline E. coli & $161(80.1)$ & $139(79.9)$ & \multirow[t]{2}{*}{.959} \\
\hline Klebsiella spp & $40(19.9)$ & $35(20.1)$ & \\
\hline \multicolumn{4}{|l|}{$\begin{array}{l}\text { Bacteremia source, } \\
\text { no. }(\%)\end{array}$} \\
\hline Urinary & $115(57.2)$ & $97(55.7)$ & \\
\hline Abdominal & $36(17.9)$ & $37(21.3)$ & \\
\hline Unknown & $25(12.4)$ & $23(13.2)$ & \\
\hline Central line & $12(6)$ & $4(2.3)$ & \\
\hline Genital & $5(2.5)$ & $2(1.1)$ & \\
\hline Pulmonary & $4(2)$ & $2(1.1)$ & \\
\hline MSK & $2(1)$ & $2(1.1)$ & \\
\hline SST & $2(1)$ & $6(3.4)$ & \\
\hline Cardiovascular & 0 & $1(0.6)$ & \\
\hline $\begin{array}{l}\text { De-escalation from } \\
\text { PT, no. (\%) }\end{array}$ & $174(86.6)$ & $155(89.1)$ & .459 \\
\hline $\begin{array}{l}\text { Duration of PT, } \\
\text { median } \mathrm{h}\end{array}$ & 83.9 & 76.2 & .68 \\
\hline LOS, median d & 16.7 & 14.8 & .373 \\
\hline AKI, no. (\%) & $37(18.4)$ & $30(17.2)$ & .769 \\
\hline CDI no. (\%) & $3(1.5)$ & $5(2.9)$ & .48 \\
\hline
\end{tabular}

Note. E. coli, Escherichia coli; MSK, musculoskeletal; SST, skin and soft tissue; PT, piperacillin/ tazobactam; LOS, length of hospital stay; AKI, acute kidney injury; CDI, Clostridium difficile infection.

analysis was completed using SPSS version 25.0 (IBM, Armonk, $\mathrm{NY}$ ). The study was approved by the institutional review board, and a waiver of informed consent was granted.

\section{Results}

The study included 201 patients in the preintervention period (March 7, 2016, through September 6, 2016) and 174 patients in the postintervention period (September 7, 2016, through March $7,2017)$. We detected no statistically significant difference between the groups in terms of age, sex, or pathogen identified. The sources of bacteremia were similar in both groups (Table 1).

Following the intervention, we detected a slightly higher de-escalation rate from PT $(89.1 \%$ vs $86.6 \%)$, although there was no statistically significant difference between the cohorts (95\% confidence interval $[\mathrm{CI}],-4.7 \%$ to $9.7 \% ; P=0.459$ ) (Table 1 ). Similarly, median duration of PT use and LOS were not significantly different before versus after the intervention: 65.3 hours (IQR, 52.9-89.2) versus
65.6 hours (IQR, 53.8-82.2) $(P=.68)$ and 7 days (IQR, 4-14) versus 6 days (IQR, $4-13)(P=.37)$, respectively. Ceftriaxone and oral ciprofloxacin were the antibiotics most commonly selected for de-escalation in both groups. The rates of AKI were similar (18.4\% vs $17.2 \% ; P$ $=.77$ ) and the number of CDI cases was low overall (Table 1).

\section{Discussion}

Few published studies have reported the effects of selective reporting on antibiotic prescribing practices, patient outcomes, and susceptibility rates. Johnson et $\mathrm{al}^{6}$ found that selective reporting implemented for cefazolin-susceptible gram-negative organisms resulted in higher rates of de-escalation from broad-spectrum $\beta$-lactams within 48 hours of release of the final results ( $71 \%$ vs $48 \% ; P=.043)$. In a Canadian study in which ciprofloxacin susceptibility was suppressed for Enterobacterales susceptible to other antibiotics, the mean monthly ciprofloxican utilization decreased from 87 to 39 defined daily doses per 1,000 patient days $(P<.001)$ with a sustained reduction in ciprofloxacin use for up to 24 months after the intervention. ${ }^{7}$ Liao et $\mathrm{al}^{8}$ assessed the effect of selective reporting on mean days of therapy of cefepime with a cascading scheme hiding cefepime susceptibilities excepting for when isolates showed ceftriaxone resistance at a break point of $\leq 8 \mu \mathrm{g} / \mathrm{mL}$. Mean days of therapy of cefepime among all patients receiving antibiotics decreased from $1.229 \pm 0.113$ before cascade to $0.813 \pm 0.813$ after cascade $(P<.0001)$. However, no differences were seen in mortality and readmissions rates.

Despite the findings of these studies, our study showed that selective reporting did not significantly affect rates of de-escalation from PT, duration of PT use, LOS, or rates of AKI and CDI. However, this finding may be due to the high baseline rate of de-escalation (86.6\%) prior to implementation of selective reporting at our institution. These findings affirm our prior evaluation of the impact of the same selective reporting on de-escalation of $\mathrm{PT}$ in patients with monomicrobial bacteremia secondary to a urinary source at the same institution. ${ }^{9}$

The strengths of this study include the relatively large cohort of patients with monomicrobial bacteremias from various of sources. Moreover, this all-inclusive study was conducted throughout a large county hospital with diverse patient and provider populations. Prescribing practices of providers from all nonpediatric departments throughout the hospital were evaluated. Additionally, no education was done prior to or during the implementation of the selective reporting change, making the study applicable to real-life situations where members of the stewardship team may not be able to provide real-time education or feedback to providers.

This study has several limitations. We did not make adjustments for potential cofounders such as comorbidities, illness severity, or hospitalization. Patients who have multiple comorbidities or are critically ill might receive longer durations of broad-spectrum antibiotics prior to de-escalation. Moreover, source control of the etiology of bacteremia was not investigated. Additionally, this intervention was intentionally not coupled with provider education. Implementation of a multimodal intervention may have resulted in a greater decrease in PT utilization and may have improved the overall impact of selective reporting given that multifaceted interventions have been shown to be highly effective. ${ }^{10}$

In this study, selective reporting of antibiotic susceptibilities did not have a significant impact on de-escalation from PT, duration of PT use, LOS, or rates of AKI and CDI. More research is needed to investigate opportunities for a standardized efficient and effective selective reporting framework. In addition, this intervention should likely be partnered with education and feedback to maximize the impact. 


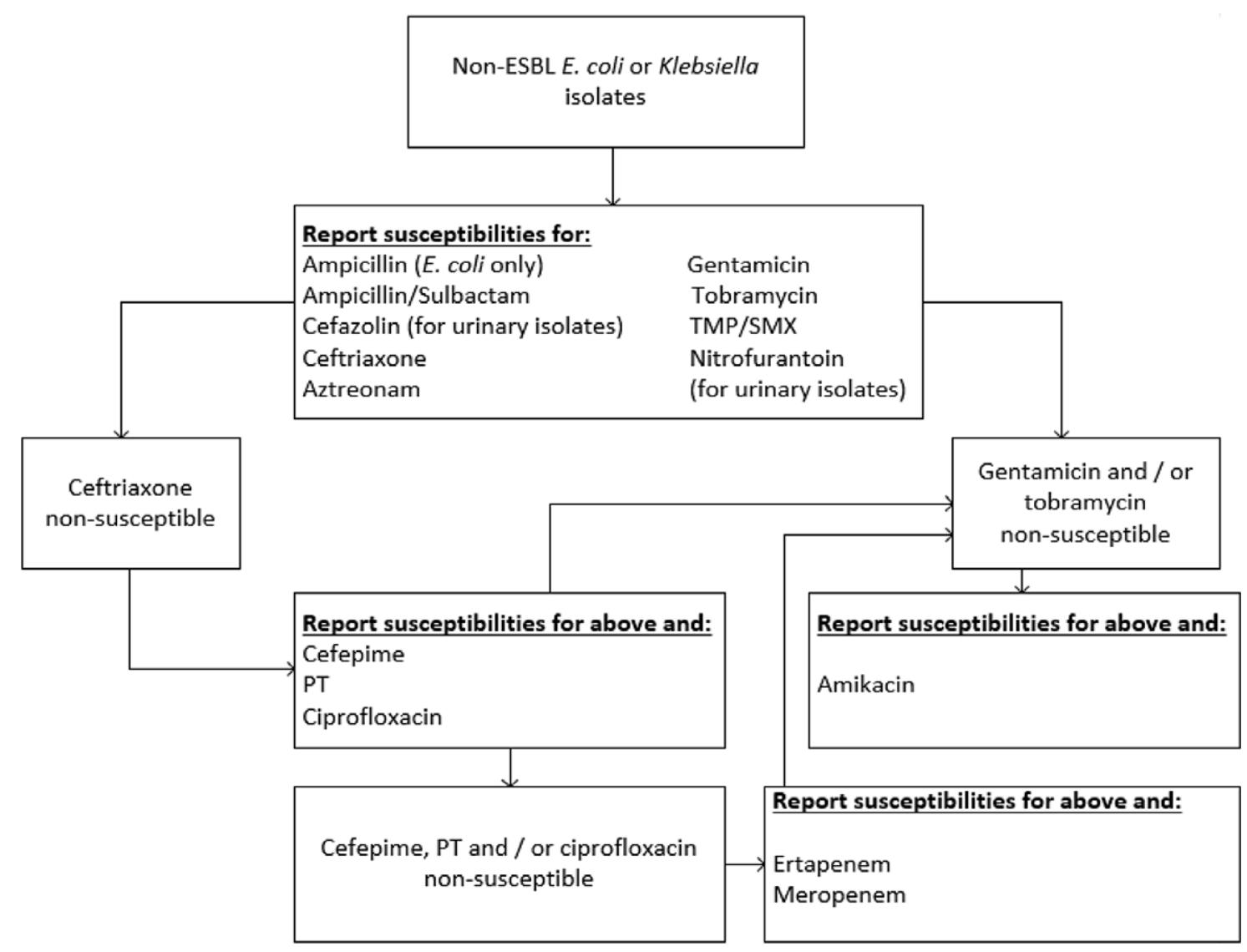

Fig. 1. Selective susceptibility reporting algorithm for E. coli and Klebsiella organisms. Note. ESBL, extended-spectrum $\beta$-lactamase; E. coli, Escherichia coli; TMP/SMX, trimethoprim/sulfamethoxazole; PT, piperacillin/tazobactam.

Acknowledgments. We acknowledge Donna L. Gaffney, MT(ASCP)SM, for her assistance with microbiology laboratory data and Debra Grant, MT(ASCP)SM, for her assistance with building the cascade logic.

Financial support. No financial support was provided relevant to this article.

Conflicts of interest. All authors report no conflicts of interest relevant to this article.

\section{References}

1. Antibiotic resistance threats in the United States, 2019. Centers for Disease Control and Prevention website. https://www.cdc.gov/drugresistance/pdf/ threats-report/2019-ar-threats-report-508.pdf. Published 2019. Accessed May 10, 2020.

2. Mauldin PD, Salgado CD, Hansen IS, Durup DT, Bosso JA. Attributable hospital cost and length of stay associated with health care-associated infections caused by antibiotic-resistant gram-negative bacteria. Antimicrob Agents Chemother 2010;54:109-115.

3. Tebano G, Mouelhi Y, Zanichelli V, et al. Selective reporting of antibiotic susceptibility testing results: a promising antibiotic stewardship tool. Expert Rev Anti-infect 2020;18:251-262.

4. Barlam TF, Cosgrove SE, Abbo LM, et al. Implementing an antibiotic stewardship program: guidelines by the Infectious Diseases Society of America and the Society for Healthcare Epidemiology of America. Clin Infect Dis 2016;62:e51-e77.

5. Lopes JA, Jorge S. The RIFLE and AKIN classifications for acute kidney injury: a critical and comprehensive review. Clin Kidney J 2013;6:8-14.

6. Johnson LS, Patel D, King EA, Maslow JN. Impact of microbiology cascade reporting on antibiotic de-escalation in cefazolin-susceptible gram-negative bacteria. Eur J Clin Microbiol Infect Dis 2016; 35:1151-1157.

7. Langford BJ, Seah J, Chan A, Downing M, Johnstone J, Matukas LM. Antimicrobial stewardship in the microbiology laboratory: impact of selective susceptibility reporting on ciprofloxacin utilization and susceptibility of gram-negative isolates to ciprofloxacin in a hospital setting. J Clin Microbiol 2016;54:2343-2347.

8. Liao S, Rhodes J, Jandarov R, DeVore Z, Sopirala, MM. Out of sight—out of mind: impact of cascade reporting on antimicrobial usage. Open Forum Infect Dis 2020;7(2):ofaa002.

9. Hall B, Alexander J, Anderson S, et al. Limited impact of selective susceptibility reporting of Escherichia coli and Klebsiella isolates from concurrent blood and urine cultures. Infect Control Hosp Epidemiol 2020. doi: 10.1017/ ice.2020.308.

10. Slain D, Sarwari AR, Petros KO, et al. Impact of a multimodal antimicrobial stewardship program on pseudomonas aeruginosa susceptibility and antimicrobial use in the intensive care unit setting. Crit Care Res Pract 2011;2011:416-426. 